\title{
Disordered peptide chains in an $\alpha$-C-based coarse-grained model
}

\author{
Łukasz Mioduszewski and Marek Cieplak* \\ Institute of Physics, Polish Academy of Sciences, \\ Al. Lotników 32/46, 02-668 Warsaw, \\ Poland
}

(Dated: July 23, 2018)

\begin{abstract}
We construct a one-bead-per-residue coarse-grained dynamical model to describe intrinsically disordered proteins at significantly longer timescales than in the all-atom models. In this model, the inter-residue contacts form and disappear during the course of the time evolution. The contacts may arise between the sidechains, the backbones and the sidechains and backbones of the interacting residues. The model yields results that are consistent with many all-atom and experimental data on these systems. We demonstrate that the geometrical properties of various homopeptides differ substantially in this model. In particular, the average radius of gyration scales with the sequence length in a residue-dependent manner.
\end{abstract}

\section{INTRODUCTION}

Coarse-grained models $1-\underline{\underline{6}}$ have provided valuable computational tools to gain insights into the temperature- or force-induced dynamics of large conformational transformations of proteins. These models have been applied mostly to proteins that are natively structured, usually globular, and they often consider the solvent to be implicit. One convenient way to implement coarse-graining is to adopt the structure-based approach ${ }^{7-11}$ in which the dynamics are governed by contact interactions and the parameters involved are derived so that the native state coincides with the ground state of the system. This idea embodies the principle of minimal frustration and leads to an optimal folding funnel $\underline{12,13}$

The issues of conformational dynamics become center-stage when describing intrinsically disordered proteins (IDPs) $\stackrel{14-18}{=}$ This is because such systems evolve through various competing basins of attraction instead of staying mostly within one deep valley in the free energy landscape, corresponding to the native state. Some IDP systems,

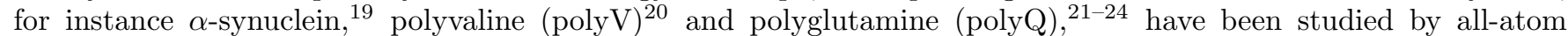
simulations, despite persistent questions about the validity of the standard force fields in this case $\stackrel{25}{2}-27^{27}$ However, the all-atom studies come with limited time-scales of the simulations and thus with restricted probing of the possible basins (this problem can be alleviated somewhat by eliminating the explicit solvent, by using GPU clusters and by shortening folding trajectories for structured proteins by involving effective polarizable force fields $\stackrel{28}{2}$ In addition, it is likely that on very long time scales many of the all-atom details of description cease to be relevant. Thus there is a need to develop coarse-grained models for molecular dynamics of the IDPs.

The existing approaches are based on the Monte Carlo method, $\underline{\underline{6}}$ Brownian dynamics,, 29 discontinuous molecular dynamics 30,31 and molecular dynamics with an explicit solvent $\underline{32}$ All of them use more than one pseudo-atom per amino acid. In addition, with the exception of the last approach, they cannot be easily applied in situations in which a protein is partially structured and partially disordered. Also, the existing hybrid Go-models with nonnative $\operatorname{contacts}^{33-35}$ do not take into account directionality and distance dependence of the contacts.

Here, we construct a novel one-bead-per-residue top-down approach formulated in the spirit of the structurebased models - specifically they involve contact interactions. The difference, however, is that the parameters used are not determined for one specific protein, but by a statistical method, based on a non-redundant set of structures from Protein Data Bank as collected in the CATH database ${ }^{36}$ The parameters, especially the values of characteristic lengths in the contact potentials, incorporate amino acid specificity. Another difference is that the establishment of contacts between the $\alpha$-C atoms is governed not only by their mutual distance but also by the expected directionality of the side chains as derived from the positions of the $\alpha$-C atoms. In this respect, we borrow from several Monte Carlo studies pertaining to the natively structured proteins $\underline{37-39}$ and fibril formation ${ }^{40}$ but provide a molecular dynamics implementation of this concept. It should be pointed out that, unlike most other coarse-grained approaches, the nature and characteristics of a given contact may be time-dependent

\footnotetext{
* E-mail: mc@ifpan.edu.pl
} 
because an interaction between, say, two sidechains may switch to that between one sidechain and the other backbone.

We show that, despite the rather approximate nature of our model, the approach yields results which are consistent with many previous computational and experimental results pertaining to the average geometry of the polypeptide chains of various lengths, $n$. We also predict values of the geometrical parameters for 20 homopeptides of the same chain length $(n=30)$ and demonstrate existence of substantial differences between the homopeptides made of different residues. We have found that in the UniProt Knowledge Database, $\stackrel{41}{\sim}$ the naturally occurring homopolymeric tracts range in the maximal sequential length, $n_{\max }$, between 6 (Ile, Tyr, Trp) and 79 (Gln). The full list of these maximal lengths is given as one of the entries in Table I Nevertheless, arbitrary lengths can be considered theoretically which allows us to determine the scaling exponents for the variations of the average radii of gyration with $n$ and show that they depend on the nature of the residue. It is expected that a chemical synthesis can lead to the tract lengths that exceed $n_{\max }$.

In this work, we consider only the case of monomers. However, the true strength of this coarse-grained approach is expected to show in studies of many-chain disordered systems such as gluten 42,43 and of large protein aggregates and complexes with disordered linkers such as arising in cellulosomes. ${ }^{4-46}$ These large systems are inaccessible to meaningful all-atom modelling and assessing, say, the viscoelastic properties of gluten requires very long simulation times.

\section{METHODOLOGY}

Our coarse-grained approach is, in many ways, as outlined in our structure-based model ${ }^{47}-49$ except that now there is no single native structure that could be used to derive parameters in the potentials. The peptide chains are represented by harmonically connected beads that also participate in transient contact interactions. The beads are located at the $\alpha$-C atoms that are at the distance, $r_{b}=3.8 \AA$ apart. The contact interactions between beads $i$ and $j$, separated by the distance of $r_{i, j}$, are described by the Lennard-Jones (LJ) potential $V_{L-J}\left(r_{i, j}\right)=4 \epsilon\left[\left(\frac{\sigma_{i, j}}{r_{i, j}}\right)^{12}-\left(\frac{\sigma_{i, j}}{r_{i, j}}\right)^{6}\right]$, where the energy parameter $\epsilon$ is assumed to be of the same order as for the structured proteins: about 110 pN $\AA$ obtained by matching to the experimental data on stretching. ${ }^{47}$ This value is close to $1.5 \mathrm{kcal} / \mathrm{mol}$ obtained independently by matching all-atom energies to the coarse-grained expressions .50 We determine the length parameter $\sigma_{i, j}=r_{\min } \cdot(0.5)^{\frac{1}{6}}$, (where $r_{\min }$ is the distance where the potential acquires its minimal value) based on the chemical identity of the residues involved. Its choice and the criteria for the contact formation will be discussed below. The elastic constant in the tethering harmonic potential, mimicking the peptide bonds, is set to $k=100 \AA^{-2} \cdot \epsilon$.

\section{A. Contact interactions}

In structure-based coarse-grained models, the contact map is fixed and is determined by the native structure. All of these contacts are occupied only in the native state, otherwise the occupation is only partial. In the case of the IDPs, there is no a priori fixed contact map. However, at each instant, there is a set of contacts that are active, i.e. they provide attraction between residues.

In order to learn how to determine which contacts are functional, we first make a survey of 21090 structured proteins from the database provided by the CATH database ${ }^{36}$ (the set of proteins with the sequence similarity not exceeding 40\%: cath-dataset-nonredundant-S40.pdb) and determine the nature of the native contacts as defined by the overlap (OV) criterion that is discussed in more details in ref 51 Briefly, the heavy atoms are represented by spheres. The radii of the spheres are equal to the context-dependent values as listed in ref ${ }^{52}$ and multiplied by 1.24 to account for attraction (the factor corresponds to the inflection point in the LJ potential) $\stackrel{53}{.}$ If at least one sphere belonging to amino acid $i$ overlaps with at least one sphere belonging to amino acid $j$ then we declare existence of the native contact between $i$ and $j$. Unlike a procedure in which a contact is declared to be present based on a cutoff distance, our criterion directly relates to the conformation of the side groups and to the sizes of the atoms.

We distinguish three classes of the contacts depending on the residue fragment from which the OV-making spheres originate: side chain - side chain (ss), backbone - backbone (bb) and backbone - side chain (bs). For a given pair of amino acids there could be simultaneous overlaps belonging to different classes. Independently of their number, only one effective contact is used in the dynamical description. It corresponds to a given $\alpha$-C $-\alpha$-C distance. We 
determine the distribution of these distances in the three classes: bb, bs and ss as illustrated in Fig. 1. However, in these distributions, the presence of, say, an ss contact does not preclude a simultaneous OV of the bs or bb kind in a given pair of residues. In our model, though, for a given distance, only one kind of contact can be operational for a pair of residues. It should be noted that our effective description involves only the $\alpha$-C atoms instead of introducing (up to) two kinds of beads to represent a residue, one for the backbone atom and another for a side chain, as proposed, for instance, by $\mathrm{Gu}$ et al $l^{\underline{54}}$

In order to associate a characteristic distance with a unique type of contact, we derive subdistributions corresponding to situations in which the OVs arise only in one class (e.g., only ss). In the case of the ss contacts, we also divide them into pairs of specific residues as listed in Table II In our model, like-charged residues do not form any ss contacts. Neither do glycine (Gly) and proline (Pro) as they can form only the backbone-involving contacts. Thus, the table has 165 entries (instead of 210).

The numbers listed in the Table M correspond to the values of $r_{\min }$ used. They range between $6.42 \AA$ (Ala-Ala) and $10.85 \AA$ (Trp-Trp). In principle, they are equal to the average values in the distribution. However, they are calculated by also taking into account additional restrictions that relate to the directionality, as discussed later on, though the corrections due to these restrictions are minor. The large value of $r_{\min }$ for the Trp-Trp pairs requires a special consideration as it would lead to unphysical extended conformations in polyW tracts that are not found in the PDB $\stackrel{55}{5}$ The longest polyW tract with a known structure is found in PDB:3N85. It has the length of 4 and it corresponds to a turn. The extended conformations do not arise if the $i, i+3$ ss contacts in the Trp-Trp pairs are disabled.

For the bb and bs cases, we take $r_{\min }$ of 5.0 and $6.8 \AA$, respectively, without incorporating any specificity.

Following previous works $\stackrel{35,40}{=}$ the bb contacts are treated in a special way: their well energy is set to $2 \epsilon$ (energy of every other contact is $\epsilon$ ) and they cannot be formed between the $i, i+4$ pairs of residues. This approach takes into account ,renumbering” $\underline{56}$ of the backbone hydrogen bonds in a coarse-grained representation of an $\alpha$-helix: the $i, i+4 \mathrm{bb}$ contacts that have been identified by the OV criterion, applied to globular structures, have the $\alpha$-C $-\alpha$-C distances that are close to $6 \AA$ (data not shown) and over $98 \%$ of them are accompanied by a simultaneously arising bs contact. Therefore, it is sufficient to attribute the effective $i, i+4$ interaction to the bs-based contact. In all other bb contacts, the typical distance is about $5 \AA$ and an association with a bs contact is much less common. Doubling the strength of the bb contacts compensates for the strict criteria for forming them, agrees with the earlier literature findings $\frac{35}{5}$ and conserves the balance between the number of each type of contacts by making the bb contacts harder to break by thermal fluctuations (in structured proteins there are about twice as many ss contacts as the bb contacts) $\underline{38}$

The time evolution is based on the equations of motion for the $\alpha$-C atoms. During its course, an effective contact may appear or disappear based on the distance between the $\alpha$-C atoms (as illustrated in Fig. 2) and on the shape of the conformation. Switching the contacts on and off is done quasi-adiabatically.

At any instant, a contact between a given pair of residues is allowed to arise only as a result of one mechanism, corresponding to its specific value of $r_{\min }$. A new type of mechanism is allowed to act only after the potential associated with the previous one drops to zero. Thus before a new type of contact can be turned on, the previous type is completely turned off. In practice, dynamical switching between various contact mechanisms occurs infrequently. The electrostatic interactions are not considered to be contacts in the sense used in our molecular dynamics model. It should be noted that if one uses the OV criterion, then the various kinds of the overlap may act together but - in the structure-based model - this results just in single standard values of the energy and length parameters of the contact, independent of the number of the contributing OVs. Thus, in our dynamical model two mechanisms cannot act together so that the contact does not get described by a sum of, say, two potentials. However, it might be interesting to consider a model in which the effective $r_{\min }$ is a weighted average of the length parameters corresponding to different successive mechanisms.

All beads are endowed with the repulsive potential to ensure that the chain never passes through itself. This potential is always turned on, regardless of the possible additional presence of the attractive potentials. The repulsion is described by the LJ potential truncated at $r_{\min }$ of $5 \AA$. For globular proteins $\frac{48}{6}$ the commonly used value is $4 \AA$, but the less constraining potentials appearing in our description of the IDPs, and hence an enhanced flexibility of the chain, require boosting the effective backbone stiffness by making the excluded volume larger.

There are several conditions that need to be satisfied in order to establish a contact. The first of these pertains to the distance, $r_{o n}$, at which the LJ potential between two beeds is switched on in addition to the repulsion. We set $r_{o n}$ to be equal to $r_{\min }$ for any given type of a contact. The other conditions will be specified in the next two 
subsections. An established contact is switched off if the $\alpha-\mathrm{C}_{i}-\alpha-\mathrm{C}_{j}$ distance exceeds $1.5 \sigma_{i, j}$.

\section{B. Effects of the directionality}

A bb contact may arise if the $\mathrm{N}$-atom on the backbone part of the $i$ th residue can form the hydrogen bond with the O-atom on the backbone part of residue $j$ or vice versa. This is allowed only if the two atoms point towards each other. This means that the vector from $\alpha-\mathrm{C}$ to $\mathrm{O}$ in one residue should be approximately antiparallel to the vector from $\alpha-\mathrm{C}$ to $\mathrm{N}$ in another. In addition, the lines set by these two vectors should nearly coincide (instead of being far away). In order to capture this physics by involving only the $\alpha$-C atoms, we use the $\mathbf{h}_{i}$ introduced in references ${ }^{37.39}$ that starts from the $\alpha$-C atom on residue $i$ and is perpendicular to the plane that is set by sites $i-1$, $i$ and $i+1$, i.e. parallel to $\mathbf{v}_{i} \times \mathbf{v}_{i+1}$, where $\mathbf{v}_{i}=\mathbf{r}_{i}-\mathbf{r}_{i-1}$ and $\mathbf{r}_{i, j}=\mathbf{r}_{j}-\mathbf{r}_{i}$. Examples of these vectors are shown on the bottom panel of Fig. 2. It can be seen that they should be lying along nearly parallel (or antiparallel, e.g. in antiparallel $\beta$-sheets) directions. This is captured by the three directional conditions imposed on the formation of an bb contact ${ }^{39}$ : a) $\left|\cos \left(\mathbf{h}_{i}, \mathbf{r}_{i, j}\right)\right|>0.92$ (the threshold angle of $\left.23^{\circ}\right)$, b) $\left|\cos \left(\mathbf{h}_{j}, \mathbf{r}_{i, j}\right)\right|>0.92$ and c) $\left|\cos \left(\mathbf{h}_{i}, \mathbf{h}_{j}\right)\right|>0.75$ (the treshold angle of $41^{\circ}$ ). The values of the thresholds have been obtained from statistical distributions of these angles 39

Defining the directionality associated with the ss contacts can be accomplished by introducing the normal vector ${ }^{40}$

$$
\mathbf{n}_{i}=\frac{\mathbf{r}_{i-1}+\mathbf{r}_{i+1}-2 \mathbf{r}_{i}}{\left|\mathbf{r}_{i-1}+\mathbf{r}_{i+1}-2 \mathbf{r}_{i}\right|}
$$

The negative normal direction $(-\mathbf{n})$ approximately points from the $\alpha$-C to $\beta$-C atoms (see the top panel of Fig. 2). A more accurate, but also more complicated, statistical expression for the location of the $\beta$-C atom can be found in the references $\frac{57.58}{3}$ However, this more accurate description is valid for $82 \%$ of the ss contacts determined by the OV criterion, while the simpler description is valid for an even higher value of 97\%. For an ss contact to form, it is necessary that the side chains point at each other. There are two conditions for this to happen 40 : 1) $\cos \left(\mathbf{n}_{i}, \mathbf{r}_{i, j}\right)<0.5$ and 2) $\cos \left(\mathbf{n}_{j}, \mathbf{r}_{j, i}\right)<0.5$ (the threshold angle of $\left.60^{\circ}\right)$. The conditions for the ss contact are qualitatively distinct from those for the bb contact because the nature of the ss contacts is much more diverse (resulting also in less restrictive thresholds), whereas the bb contacts have a specific hydrogen bonding pattern. 39

Similar considerations lead to the following two conditions for a bs contact to form (the sidechain of the $i$ th residue interacts with the backbone of the $j$ th residue, see the middle of Fig. 20): 1) $\cos \left(\mathbf{n}_{i}, \mathbf{r}_{i, j}\right)<0.5$ and 2 ) $\left|\cos \left(\mathbf{h}_{j}, \mathbf{r}_{j, i}\right)\right|>0.92$.

As demonstrated in Fig. 1, most of the OV-based contacts in the native states of the structured proteins satisfy the directional criteria as listed above. Exceptions occur mainly in the bs contacts, where the nature of the contact may not be fully captured by directional criteria associated separately with the ss and bb cases. The number of contacts formed between the backbone and hydrophobic sidechains is comparable to those formed with polar sidechains. Thus the nature of the bs overlap contacts can be difficult to describe and the corresponding characteristic distances are much less specific than those corresponding to the ss contacts. Imposing directional criteria shifts $r_{\text {min }}$ of the bs contacts from 6.8 Ato $5.4 \AA$, but this does not change the results in any significant manner.

Acceptable values of $\cos \left(\mathbf{n}_{i}, \mathbf{r}_{i, j}\right)$ seem to be largely independent from the $r_{i, j}$ distance itself. Examples of distributions on the plane corresponding to $\cos \left(\mathbf{n}_{i}, \mathbf{r}_{i, j}\right)$ and $r_{i, j}$ are shown in Fig. S4 in the Electronic Supplementary Information (ESI). We do not introduce any potentials that would favor the directionalities to stay within the appropriate bounds - they are checked only at the instant when the contact is established.

\section{The types and specifity of effective residues}

We group the residues into six classes: 1) Gly, 2) Pro, 3) hydrophobic: Ala, Cys, Val, Ile, Leu, Met, Phe, Tyr, Trp, 4) polar: Gln, Ser, Thr, Asn, His 5) negatively charged: Asp, Glu, and 6) positively charged: Arg, Lys (see Table I). The last two classes count as polar but they also carry charge. The distinction between the polar and hydrophobic residues matches (with the exception of Ala) the clustering (based on the eigenvalue decomposition) 
of the Miyazawa-Jernigan interaction matrix into two clusters $\underline{59}$ Contacts of the ss kind can be made between the hydrophobic residues $\stackrel{40}{=}$ but also between the polar ones (crucial for modeling of polyQ) and between the polar and hydrophobic residues. The unlike-charged polar residues may form salt bridges but the like-charged ones merely repel. The energy parameter for each type of the ss contacts is the same. This, together with allowing for the formation of hydrophobic-polar ss contacts, distinguishes our model from the simple „HP” models, $\underline{40}$ where such contacts either have reduced strength or are not allowed to arise. Thus, even though our model cannot capture the details of all possible interactions (for instance, between the $\pi$ electrons on the faces of aromatic rings and cations), our models allows for them statistically.

Real cysteine residues may form disulfide bonds, but this process is not covered in this paper, as none of the considered systems seems to form such bonds. They can be implemented in a coarse-grained model as discussed in references $\underline{60,61}$

Each residue is allowed to form up to $z_{s}$ contacts with other residues. The number $z_{s}$ is residue-dependent. We take $z_{s}=n_{b}+\min \left(s, n_{H}+n_{P}\right)$, where $n_{b}$ is the permitted number of backbone contacts, $s$ is the maximum allowed number of the sidechain contacts and $n_{H}, n_{P}$ are the maximal numbers of sidechain contacts with hydrophobic and polar sidechains of other residues, respectively. The values of these parameters are listed in Table I For a bs contact, one backbone "slot" assigned to one residue must be combined with one sidechain "slot" assigned to the other residue. The backbone does not count as polar in bs contacts, so it affects only the total $s$ limit for a given sidechain. Treating the backbone as a polar entity merely changes the proportions between the types of contacts arising without improving the results. $n_{b}$ is equal to 2, corresponding to two possible hydrogen bonds. In the case of Pro, $n_{b}=1$.

The numbers $n_{H}, n_{P}$ and $s$ depend on the type of the residue and were derived from the structure database by calculating, for each residue of a given type, the distribution of its observed numbers, $s_{c}$, of the ss contacts, i.e. the coordination numbers. $s$ was operationally defined in relation to $s_{\max }$ - the values of $s_{c}$ corresponding to the maxima in such distributions. For the hydrophobic residues, the distributions are broad and we take $s=s_{\max }$. For the polar residues, the distributions are narrow and then we take $s=s_{\max }+1$. The polar residues usually stay near the surface of a protein and thus some of their possible 'contacts' are with the molecules of water instead of with the other residues and hence the under-count in the distribution. This is also the reason why we do not determine $s$ by counting the possible hydrogen bonds that could arise. The values of $n_{H}, n_{P}$ were determined similarly, by calculating two-dimensional distributions of pairs of numbers of hydrophobic and polar contacts (with maxima at points $\left.s_{H \max }, s_{P \max }\right)$. Then, $n_{H}$ corresponds to the maximum $s_{H \max }$ of such distribution (the most common number of hydrophobic ss contacts for a given type of amino acid), $n_{P}$ to $s_{P \max }+1$. The values of $s, n_{H}, n_{P}$ for the different types of residues are listed in Table [.

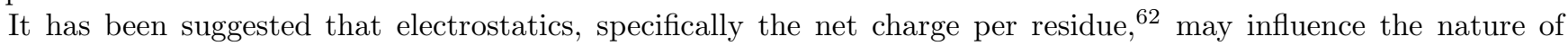
conformational ensembles of the IDPs substantially. Thus, in addition to the ss, bb, and bs contacs, the charged residues interact with each other via the modified Debye-Hueckel potential ${ }^{63}$

$$
V_{D-H}=\frac{e^{2} \exp (-r / \lambda)}{4 \pi \varkappa \varkappa_{0} r}
$$

with the screening length $\lambda=10 \AA$. Following Tozzini et al.,,$\frac{5}{,}$ the relative permittivity is taken to be $\varkappa=4 \AA^{-1} r$ (generally, $\varkappa$ changes with the distance sigmoidally so that it is around 4 well within the protein and around 80 at large distances; $\varkappa_{0}$ is the permittivity constant). This leads to the effective electrostatic potential which is approximately equal to $V_{e l}(r)=\frac{85 \exp (-r / \lambda) \epsilon \AA^{2}}{r^{2}}$. This long-range interaction does not count into the $z_{s}$ limit. When two oppositely charged residues form an ss contact, their interaction becomes described by the standard LJ contact potential with $r_{\min }$ taken from Table II in an analogy to the way the salt bridges are described in the structure-based models of the structured proteins. When a salt bridge is adiabatically turned on, other electrostatic interactions made by these two residues are turned off in the same manner, so that the local geometry of the bridge is stable. However, we have found that this precaution does not affect the results noticeably. Like-charged residues cannot form the ss contacts but they may participate in the bb contacts. This is possible for certain conformational geometries or for situations in which at least one of the charges is effectively removed due to the formation of the ionic bridge with another residue. The first and last residues in the chain are not allowed to form attractive inter-terminal LJ contacts so that the protein does not form a closed loop (in Enciso and Rey's model $\underline{39}$ the inter-terminal contacts may exist, but their strength was reduced). In an all-atom model, the termini do come with opposite electric charges but this is usually not so in coarse-grained models in which the terminal residua have the same properties as the non-terminal ones. 


\section{The backbone stiffness}

The backbone stiffness is usually defined in terms of bond and dihedral angles. In structure-based models of proteins, these angles can be measured as deviations from their local native values. In the IDP case there are no such reference values and we resort to a statistical approach as proposed by Ghavani et al . $^{64}$

In this approach, and when determining the distribution of bond angles, one divides the residues into three types: Gly, Pro and X, where X denotes all other 18 residues. There are 27 possible combinations of these residues and the bond angle associated with the middle of the three consecutive residues is governed by a distribution specific to the triplet. The distributions have been obtained by matching to the experimental Ramachandran angles 64 and are found to be fairly broad: they typically span about $60^{\circ}$ whereas the structure-based potentials are significantly narrower. We use the potentials that distinguish only the middle residue and whether it precedes proline, which results in dealing with only 6 possible combinations. The sequential order of the residues matters because it defines the local chirality. Thus we can only distinguish cases in which a proline is preceded but not when it is succeeded. The examples of statistical potentials are shown in Fig. S5 in ESI. In our molecular dynamics simulations, we fit each statistical potential to a sixth degree polynomial with the coefficients listed in Table S1 in ESI.

The dihedral angle requires a consideration of four consecutive residues that can be viewed as a superposition of two triplets that overlap at two central residues. In the three-alphabet case, there are 9 possible overlaps and thus 9 distributions. We show examples of the statistical potentials for these distributions in Fig. S6 in ESI. We fit the statistical potentials to the formula: $a \sin (x)+b \cos (x)+c \sin ^{2}(x)+d \cos ^{2}(x)+e \sin (x) \cos (x)$, where the values of the coefficients are also listed in Table S2 in ESI.

Both of the bond-angle and the dihedral potentials were obtained by the inverse Boltzmann method at the room temperature applied to the random coil database statistics. These potentials correctly describe properties of denaturated proteins,$\stackrel{64}{=}$ that can be assumed to have no contacts. A priori, the energy scale involved may not exactly match the one associated with the contact energy. We have checked, however, that doubling the amplitudes of the angle-involving potentials does not affect the geometrical parameters of the test systems in any significant manner.

\section{E. The molecular dynamics simulations}

The solvent is implicit. The time evolution is defined in terms of molecular dynamics with the velocity-dependent damping and the Langevin noise, both representing the influence of the solvent. The noise corresponds to temperature $T$. The characteristic timescale, $\tau$ is of order $1 \mathrm{~ns}$ since the system is considered to be overdamped so that the motion of the beads is diffusional instead of ballistic. The damping constant, $\gamma$, is taken to be $2 m / \tau$, where $m$ is the typical mass of a residue. More realistic values of $\gamma$ should be about 25 times larger $\underline{65}$ but adopting them would lead to much longer conformational dynamic.

As described earlier, there are three types of requirements for a contact to be legitimate: 1) on the distance between the residues, 2) on the proper directional placement of the implicit side groups and backbone hydrogen bonds, 3) on the number of contacts that the residues are permitted to form. If all of these requirements are fulfilled, a contact is made. A sudden creation of a contact may lead to instabilities and hence we switch the corresponding contact potential on adiabatically: the depth of the potential well increases linearly form 0 to $\epsilon$ within the timescale of $10 \tau$. This timescale is sufficiently long for the system to thermalize so the process is quasi-adiabatic. We have observed that shorter switching-on times lead to heating of the system (see the top panel of Fig. S7 in ESI). Longer times, up to $40 \tau$, do not affect the statistical properties that we measure. Still longer times make the chains more mobile which shows as a slight expansion of the system (bottom panel of Fig. S7 in ESI). Increasing $\gamma$ is expected to lead to more stable dynamics and longer permissible switching-on times.

We integrate the equations of motion by the fifth order predictor-corrector algorithm ${ }^{66}$ and each $\tau$ is discretized into 200 steps. Thus the adiabatic switching-on process is accomplished in 2000 steps. Switching off of the contacts, when the contact distance crosses $1.5 \sigma_{i, j}$, is implemented with the same time scale. If a pair of residues is connected by a contact of one type, it cannot be simultaneously connected by a contact of another type.

As explained in reference $\frac{67}{}$ the kinetic and thermodynamic behavior of a coarse-grained model depends on the type of the backbone stiffness used. The $T$-range in which folding of structured proteins is optimal is around $0.3-$ $0.35 \epsilon / k_{B}$ if the backbone stiffness is described by a chirality potential. In this case then $0.35 \epsilon / k_{B}$ corresponds to the room temperature, $T_{r}$, which is consistent with the callibrated value of $\epsilon$. However, for the bond and dihedral 
potential $T_{r}$ shifts to about $0.7 \epsilon / k_{B}$, because these terms contribute more substantially to the energy.

In order to determine $T_{r}$ for our IDP model, we perform a similar folding test for several globular proteins with the PDB structual codes of 1GB1, 1TIT, 1UBQ, and 2M7D. We take the native contacts map but adopt the statistical angular potentials for the backbone stiffness. We find that the kinetic optimality is reached around $0.3-0.35 \epsilon / k_{B}$. In addition, Fig. S8 in ESI shows that for $0.3 \epsilon / k_{B}$, the average end-to-end distance matches the room-temperature all-atom results for polyQ for $n$ up to 60 and it does not for higher temperatures. The matching at $0.2 \epsilon / k_{B}$ is comparable, but folding is significantly worse than at $0.3 \epsilon / k_{B}$ due to the emergence of more potent kinetic traps. Moreover, Fig. S8 demonstrates a similar matching to the experimental results for the flanked versions of polyQ. We have also found that best agreement with experimental data for polyproline occurs for temperatures in range 0.3 $0.4 \epsilon / k_{B}$. (results for $T=0.3 \epsilon / k_{B}$ will be discussed later). Thus, in our simulations we take $0.3 \epsilon / k_{B}$ as the $T$ for which most simulations were made.

The results on the geometry of the systems were obtained based on 100 independent trajectories, each lasting for $100000 \tau$. The time evolution in the first $5000 \tau$ was excluded from the data acquisition to allow for equilibration. The conformations were saved every $100 \tau$, unless stated otherwise. For structured proteins, we started from the native structure whereas for the IDPs - from a self-avoiding random walk.

\section{RESULTS}

The main purpose of the performed simulations is to provide a validation of our model. We use three parameters to characterize the geometrical properties of an IDP. One is the time-averaged radius of gyration $R_{g}=\sqrt{\left\langle r_{g}^{2}\right\rangle}$, where $r_{g}$ is the instantaneous value of this radius. Another is $l$ - the time averaged end-to-end instantaneous distance $d_{e e}\left(l=\left\langle d_{e e}\right\rangle\right)$. The third is $\sigma=\sqrt{l^{2}-\left\langle d_{e e}\right\rangle^{2}}$, which is the dispersion in $d_{e e}$. As described by Różycki et $a l .{ }^{46,68} \sigma$ can be used to define the effective elastic stiffness, $\kappa$, of the system, since energy equipartition implies that $\frac{1}{2} \kappa \sigma^{2}=\frac{3}{2} k_{B} T$. The nature of our coarse-grained approximation does not allow us to make predictions about local structural parameters such as the Ramachandran angles and thus to make the corresponding comparisons.

Generally, we test the predictions of our model against the results obtained through all-atom simulations, in which the geometrical parameters were calculated, and against the experimental results. We have chosen the NMR and SAXS experiments in which few interpretational assumptions about the parameters were made. The comparisons are shown in Figs. 4, 5, and 6. The first two of these present comparisons to the all-atom results and the last - to the experimental ones.

\section{A. PolyQ and polyV}

Fig. 4 pertains to polyQ and polyV for $n$ of up to 60 . We use the notation $\mathrm{Q}_{n}, \mathrm{~V}_{n}$, and similarly for other amino-acid homopolymers. Cossio et al. 20 have generated 30063 statistically independent conformations for $\mathrm{V}_{60}$ by using all-atom simulations in a meta-dynamics approach involving the replica-exchange method. The solvent was implicit and treated within the generalized Born surface area approach. They concluded that only a fraction of these conformations is structurally similar to the proteins listed in the CATH database. They took it as an evidence that there are evolutionary pressures that favor only selected classes of conformations. Here, we calculate $l, \sigma$, and $R_{g}$ for 7077 confromations of $\mathrm{V}_{60}$, that were made available to us by the authors, by assuming that these conformations approximately represent an equilibrium trajectory. Our model is seen to generate parameters that are larger by a factor of around 2 (depending on which quantity is considered) for $n=60$, but the differences are expected to get smaller for lower values of $n$.

It should be noted that $\mathrm{V}_{n}$ does not exist in nature whereas long tracts of polyQ do arise in various proteins, for instance in the context of Huntington disease. Gómez-Sicilia et al. ${ }^{24}$ have applied the approach of Cossio et al. ${ }^{20}$ to $\mathrm{Q}_{n}$ with $n$ between 16 and 80. The statistics of the independent conformations were 308, 491, 330, 422, 479, 322, 269,246 , and 108 for $n=16,20,25,30,33,38,40,60$, and 80 respectively. We do not consider $n=80$ here due to the smaller statistics. 
Fig. 4 demonstrates that the geometrical parameters, calculated based on the statisticaly independent conformations obtained by Gómez-Sicilia et al $\underline{24}^{24}$ agree fairly well with the results of our model, especially in the case of the parameter $l$. Generally, the smaller the $n$, the closer the agreement, especially in the case of $l$. We consider the agreement to be adequate enough to model gluten (under study) which consists of many Q-rich chains $\underline{\underline{43}}$ We have observed that a better agreement with the all-atom data on polyQ can be obtained by increasing the value of the parameter $s$ (the number of possible sidechain contacts) from 2 to 3 . However, we keep $s=2$ in order to achieve optimal consistency for all systems considered here. Increasing the limit for backbone contacts, $n_{b}$, from 2 to 3 , results in a similar improvement for $R_{g}$ (though not for $\sigma$ ) for this system.

One of the findings of Gómez-Sicilia et al. 24 was that a substantial fraction (9.3\%) of the statistically independent conformations of $\mathrm{Q}_{60}$ are knotted and that the sequential extension of the knotted segments starts at about 36 residues. Such knotted conformations may jam the proteasome and thus lead to toxicity $\underline{69}$ In experimental systems,$\underline{70}$ the toxicity arises above a threshold of about 35. Our coarse-grained model has produced several knotted conformations for $\mathrm{Q}_{60}$ (all with the shallow knots) and none for $\mathrm{V}_{60}$. In the all-atom model, $\mathrm{V}_{60}$ had a factor of 3 smaller propensity to form knots than $\mathrm{Q}_{60}$ so there is a qualitative agreement. An example of a knotted conformation obtained for $\mathrm{Q}_{40}$ is shown in the rightmost panel of Fig. 7 Increasing $s$ from 2 to 3 has been found to enhance the probability of knotting (but still below 9\%). (We find that more substantial knotting propensities are exhibited by polyW).

Since the IDP systems flow from one conformation to another, it is interesting to ask for how long do they stay in particular conformations. One measure to assess this is to probe the system every $1 \tau$ and determine the time needed to deviate in RMSD (root mean square deviation) from the considered conformations by $5 \AA$. For $\mathrm{Q}_{60}$ the distribution of such times extends up to $65 \tau$ and has the mean of $24 \tau$ and the dispersion of $10 \tau$. A typical duration time obtained in the all-atom simulations ${ }^{24}$ is up to of order 20 ns, i.e. comparable to that obtained in the coarse-grained simulations.

Another way to characterize conformational transformations is to use the fraction, $f_{c c}$, of the contacts that are common with a reference structure. The RMSD is more related to the overall shape whereas $f_{c c}$ to the nature of the local cohesion. For instance, bending of the chain segments results in an increase in the RMSD but may leave $f_{c c}$ largely unchanged, indicating persistence of the local structure. The time dependence of the two measures for a trajectory obtained for $\mathrm{Q}_{60}$ is shown in Fig. 3 . We consider three reference structures: one (the lines in green; denoted by A) obtained at $10^{5} \tau$, another (the lines in black; denoted by B) at $210^{5} \tau$ and still another (the lines in blue) at $310^{5} \tau$. The red vertical line indicates time at which, based on $f_{c c}$, the conformation stops being more alike to conformation A and becomes more alike to conformation C. This happens at about $1.5310^{5} \tau$ indicating that the changes in the contact map take place in the scale of microseconds. Interestingly, a similar transition to conformations similar to B (the black vertical line), which is closer in time, takes place at the later instant of $1.6710^{5} \tau$. In contrast, the RMSD crosses the 5 - $\AA$ treshold much more rapidly within $0.0510^{5} \tau$. A more detailed characterization of the time evolution in terms of $f_{c c}$ can be found in ESI (Fig. S9).

Experimental FRET data for polyglutamine chains of length $m$ equal to 8, 12, 16, 20 and 24 have been obtained by Walters and Murphy $\stackrel{71}{ }$ for two values of $\mathrm{pH}: 7$ and 12 . These chains, however, are flanked by the sequence KKW on the N-terminus and by AKK on C-terminus so the backbone length $n=m+6$. Thus the model-based results require recalculations, especially because the presence of the lysines brings in electrostatic effects, providing another kind of test of the model. Fig. 5 shows our results compared to the experimental ones for $\mathrm{pH}=7$. The systems are denoted as KQmpH7 and the data points are shown in green. There is a close agreement for $l$ but a discrepancy for $\sigma$. The experimental results on $\sigma$ apper unreasonably small though, particularly at $n$ of 30 (the case shown in the figure), where the Coulomb effects should be too weak to keep the system stiff and barely fluctuating. All theoretical data points are distinct from the range of values found in systems without the flanking lysine (Fig. (4). If we assume that changing

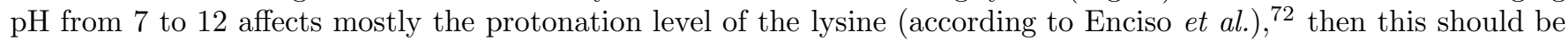
equivalent to replacing the charged lysine by the neutral asparagine. Our theoretical results with the asparagine show correlations with the experimental data at $\mathrm{pH}=12$ (see Fig. S10 in ESI) of a similar quality as with the lysine at pH=7.

\section{B. PolyA and polyP}

Another interesting system is polyA. The all-atom results, which used the TIP3P model for the molecules of water $\underline{73}$ and the CHARMM36 protein force field, have been derived $\stackrel{46}{\underline{6}}$ for $l$ and $\sigma$ and for a set of values of $n$. They are compared to our coarse-grained results in Fig. 5. We observe that there is a good agreement for small values of $n$, up to 15, when the conformations are helix-like (see also ref.) ${ }^{20}$ as illustrated in the left panel of Fig. 7 . At 
larger values of $n$, the coarse-grained data fall down relative to the all-atom ones. We attribute the discrepancy to the differences in the timescales. In the all-atom simulations it is $100 \mathrm{~ns}$ whereas in the coarse-grained model of order $5 \mu \mathrm{s}$, i.e. 50 times longer and repeated in 100 trajectories. In the latter situation, the chain can explore significantly more phase space and adopt bent conformations such as the one shown in the middle panel of Fig. 7 . Such bent conformations allow for a closer approach of the termini to each other than in the case of a helix. They have been observed in simulations with an implicit solvent ${ }^{74}$ for $n$ exceeding 40 . The threshold $n$ above which the bent conformations may appear must depend on the description of the backbone stiffness - in our case the backbone is rather soft (the constraints on the bond angle are weak) and hence the lowering of the threshold. A better modeling of the bond-angle potential should increase the treshold.

The results for polyP are shown in Fig. 6. The agreement with the FRET-based data ${ }^{75}$ on $l$ and $\sigma$ is pretty good. All three geometrical parameters exhibit a relative steep growth with $n$, reflecting the fact that the shape of the conformations is governed primarily by the backbone-stiffness potentials so that the details of any non-local potentials have only a minor impact.

\section{Other systems}

In studies on glycine-rich systems ${ }^{76.77}$ the FRET efficiency is not used to calculate $l$ directly. Instead, it serves as a constraint that is employed to reconstruct the distance distributions by using simple models. These systems incorporate repetitive sequences containing glycine (like GGS or GGGGS, collectively named „GS”). They are expected to be prone to a substantial flexibility. This expectation is confirmed (see the plot of $\sigma$ in Fig. [5). Furthermore, there is a good agreement of the model results with the FRET-based data.

We now consider the disordered cellulosomal linkers that are listed in Table 1 of reference $\underline{46}$ In order to avoid crowding in Fig. 5] we show the results only for the linkers with $n=10$ and $n \geq 17$ (the symbols in blue). The data points are scattered - however, they are comparable to the all-atom explicit-solvent simulations for these systems (there are no all-atom results for $R_{g}$ ).

IDP's may require using different force fields than the structured proteins. Huang et al ${ }^{27}$ have recently proposed a new force field, CHARMM36m, which should show an improved performance both in the IDP and structured cases. Several proteins were considered as test cases. Among them, the FG-nucleoporin and the RS peptide, both disordered. Fig. 5 shows that our model gives results that are comparable to those obtained by the novel force field ( $l$ and $\sigma$ are available for the RS peptides and $R_{g}$ for the FG-nucleoporin peptide). The agreement gets worse for Histatin-5 (His5, used to parameterize the Amber and Gromos force fields) ${ }^{78}$ probably because we consider His to be uncharged. An admixture of the charged states of His should introduce electrostatic repulsion and thus larger values of $R_{g}$.

The Protein Ensemble Databank (PE-DB $)^{79}$ contains a number of structural data which were obtained through simulations which are considered to be consistent with the ensembles obtained in the NMR and SAXS experiments data on IDP's. These proteins have $n$ considerably larger than the systems considered so far. We find (see Fig. 6) that our results on $R_{g}$, though not coinciding with the PE-DB results, are of comparable size. However, the discrepancies are larger for 6AAA and 9AAA (not shown). It should be noted, however, that the PE-DB results we compare to were obtained with the use of particular all-atom and/or coarse-grained models. In addition they were derived for a given timescale which may or may not be adequate.

\section{Comparisons of homopeptides}

Table I lists the known maximal lengths of tracts formed by amino acids of the same type, as obtained from the UniProt database. $\stackrel{41}{*}$ The longest of them correspond to Gln (79), Ser (58), and Asn (46). For Ala the length is 24 but for the sligthly larger Val - only 9 . Nevertheless, it is instructive to simulate homopeptides of unrestricted length. In particular, Cossio et al. have studied the chains of Val with $n=60$. Here, we simulate all homopeptides of length 30 to bring out the differences in behavior between them, as derived using our model. The results on the geometrical parameters are shown in Fig. 8 and on the properties of the contacts in Fig. 9. The properties of the contacts are assessed by providing the average coordination number, $\langle z\rangle$, and the average sequential distance in the contacs, 
$<|j-i|>$. The former includes the contacts made by the dipeptide bonds along the backbone. The latter, when divided by $n$, defines a parameter known as the contact order.

All of the homopeptides of length 30 appear to be IDP's since $\sigma$ is always substantial: it exceeds $5 \AA$. In agreement with Uversky ${ }^{18}$ and Mao et al. ${ }^{62}$ there is a clear difference between the charged polymers (polyK, polyE, polyR, and polyD) and the collapsed non-charged systems. The charged homopetides have the largest values of $R_{g}$ and $l$ as they do not form any contacts. PolyP comes next - these chains also do not form contacts. Due to the restricted bending capabilities, polyP is the stiffest of the homopolymers considered here (the lowest vakues of $\sigma$ ). However, the properties of polyW appear to be the most extreme: it shows the lowest values of $R_{g}$ and $l$ and the highest values of $\langle z\rangle$ and $\left\langle|j-i|>\right.$. These features result from the largest value of $r_{m i n}$ and hence from the dominance of non-local ss contacts (every W residue can form up to 4 of them). There are almost no bb contacts in the simulated polyW systems. PolyW is also the most likely to generate knotted structures. The knots appear in $16 \%$ of the trajectories and last for about $5 \%$ of total simulation time.

Other hydrophobic homopolymers are generally more compact and less flexible than the polar ones, with the exception of polyG and polyA that are endowed with short side chains. This can explain the fact that the longest hydrophobic residue tracks seen in the UniProt database are shorter than those made from the polar residues (again with the exception of polyG and polyA that are more flexible and thus more accomodating.)

Another way to compare the homopeptides is to study the scaling behavior of radius of gyration in a broad range of the values of $n$ : between 10 and 100. For homopolymers with $n \neq 30$ each MD trajectory was $50000 \tau$ long. We observe a good agreement with the power law for

$$
R_{g} \sim n^{\nu}
$$

The values of the exponent $\nu$ are listed in Table I. We find that $\nu$ 's for the large hydrophobic amino acids are smaller (which corresponds to the poor solvent regime) ${ }^{80}$ than for the polar and small hydrophobic amino acids (for which water should be better solvent). Thus our model is seen to capture the substantial differences between the residue types in the correct manner.

\section{CONCLUSIONS}

We have constructed an $\alpha$-C based molecular dynamics model of IDP which, despite its simplicity, yields structural results that are in a reasonable agreement with all-atom and experimental data and allows for an access to significantly longer timescales than in the all-atom simulations.

There is a category of IDPs that acquire structure on binding with a substrate ${ }^{81}$ Our model is likely to fail when using it to predict the specific structure that arises on binding. The model requires a more stringent fine tuning for this purpose. We have also found that the potentials used in our model cannot keep any structured proteins in their native conformation, as equilibrium inter-residue distances are different. Thus applying our model to study, say, folding of the structured proteins leads to a fairly chaotic behavior. In this case, using the structure-based models is much more effective.

An alternative way to introduce specificity into the ss contacts is to use the sums of statistically determined radii associated with the residues ${ }^{82}$ instead of the pair-wise characteristics summarized in Table II] This approach would introduce merely 20 residue-related parameters. It remains to be explored whether it provides an improvement over the methodology presented here.

Acknowledgements We appreciate fruitful discussions with B. Różycki as well as receiving help of G. Matyszczak and K. Wolek with the calculations. MC has received funding from the National Science Centre (NCN), Poland, under grant No. 2014/15/B/ST3/01905 and by the EU Joint Programme in Neurodegenerative Diseases project (JPND CD FP-688-059) through the NCN grant 2014/15/Z/NZ1/00037. He was also supported by the NCN grant No. 2016/21/B/NZ1/00006. The computer resources were supported by the PL-GRID infrastructure and also financed by the European Regional Development Fund under the Operational Programme Innovative Economy NanoFun 
POIG.02.02.00-00-025/09.

1 M. Levitt and A. Warshel, Computer simulations of protein folding. Nature, 1975, 253, 694-698.

2 M. Levitt, A simplified represntation of protein conformations for rapid simulation of protein folding. J. Mol. Biol., 1976, 104, 59-107.

3 A. Kolinski, ed., Multiscale approaches to protein modeling: structure prediction, dynamics, thermodynamics and macromolecular assemblies, Springer, New York, 2010.

4 A. Liwo, ed., Computational methods to study the structure and dynamics of biomolecules and biomolecular processes - from bioinformatics to molecular quantum mechanics, Springer, Heidelberg, 2014.

${ }^{5}$ V. Tozzini, J. Trylska, C. Chang and J. A. McCammon, Flap Opening Dynamics in HIV-1 Protease Explored with a Coarse-Grained Model. J. Struct. Biol., 2007, 157(3), 60615.

${ }^{6}$ Y. C. Kim and G. Hummer, Coarse-grained models for simulations of multiprotein complexes: application to ubiquitin binding. J. Mol. Biol., 2008, 375, 1416-1433.

7 Y. Ueda, H. Taketomi and N. Go, Studies on protein folding, unfolding and fluctuations by computer simulations. Biopolymers, 1978, 17, 1531-1548.

8 I. Shrivastava, S. Vishveshwara, M. Cieplak, A. Maritan and J. R. Banavar, Lattice model for rapidly folding protein-like heteropolymers. Proc. Natl. Acad. Sci., 1995, (USA) 92, 9206-9209.

9 N. Koga and S. Takada, Roles of native topology and chain-length scaling in protein folding: a simulation study with a Go-like model. J. Mol. Biol., 2001, 313, 171-180.

10 C. Clementi, H. Nymeyer, and J. N. Onuchic, Topological and energetic factors: what determines the structural details of the transition state ensemble and "en-route" intermediates for protein folding? An investigation of small globular proteins. J. Mol. Biol., 2000, 298, 937-953.

11 T. X. Hoang and M. Cieplak, Molecular dynamics of folding of secondary structures in Go-like models of proteins. J. Chem. Phys., 2000, 112, 6851-6862.

12 J. D. Bryngelson and P. G. Wolynes, Spin glasses and the statistical mechanics of protein folding. Proc. Natl. Acad. Sci., 1987, USA 84, 7524-7528.

13 D. Baker, A surprising simplicity to protein folding. Nature, 2000, 405, 39-42.

14 P. E. Wright and H. J. Dyson, Intrinsically unstructured proteins: Re-assessing the protein structure-function paradigm. J. Mol. Biol., 1999, 293, 321-331.

15 A. L. Fink, Natively unfolded proteins. Curr. Opin. Struct. Biol., 2005, 15, 35-41.

16 V. N. Uversky and A. K. Dunker, Understanding proteins non-folding. Biochem. Biophys. Acta, 2010, 1804, $1231-1264$.

17 A. C. M. Ferreon, C. R. Moran, Y. Gambin and A. A. Deniz, Single-molecule fluorescence studies of intrinsically disordered proteins. Methods Enzymol,, 2010, 472, 179-204.

18 V. N. Uversky, Unusual biophysics of intrinsically disordered proteins. Biochim. Biophys. Acta, 2013, 1834, $932-951$.

19 A. Sethi, J. Tian, D. M. Vu and S. Gnanakaran, Identification of minimally interacting modules in an intrinsically disordered protein. Biophys. J., 2012, 103, 748-757.

20 P. Cossio, A. Trovato, F. Pietrucci, F. Seno, A. Maritan and A. Laio, Exploring the universe of protein structures beyond the Protein Data Bank. PLoS Comp. Biol., 2010, 6, e1000957.

21 A. Vitalis, X. Wang and R. V. Pappu, Quantitative characterization of intrinsic disorder in polygultamine: Insights frm analysis based on polymer theories. Biophys. J., 2007, 93, 1923-1937.

${ }^{22}$ L. Esposito, A. Paladino, C. Pedone and L. Vitagliano, Insights into structure, stability, and toxicity of monomeric and aggregated polyglutamine models from molecular dynamics simulations. Biophys. J., 2008, 94, 4031-40.

${ }^{23}$ H. Ogawa, M. Nakano, H. Watanabe, E. B. Starikov, S. M. Rothstein and S. Tanaka, Molecular dynamics simulation study on the structural stabilities of polyglutamine peptides. Comp. Biol. Chem., 2008, 32, 102-110.

24 Á. Gómez-Sicilia, M. Sikora, M. Cieplak and M. Carrión-Vázquez, An exploration of the universe of polyglutamine structures PLoS Comp. Biol., 2015, 11, e1004541.

25 S. Rauscher, V. Gapsys, M. J. Gajda, M. Zweckstetter, B. L. de Groot and H. Grubmueller, Structural ensembles of intrinsically disordered proteins depend strongly on force field: A comparison to experiment. J. Chem. Theor. Comp., 2015, 11, 5513-5524.

${ }^{26}$ W. Wang, W. Ye, C. Jiang, R. Luo and H.-F. Chen, New force field on modeling intrinsically disordered proteins. Chem. Biol. Drug Des., 2014, 84, 253-269.

27 J. Huang, S. Rauscher, G. Nawrocki, T. Ran, M. Feig, B. L. de Groot, H. Grubmueller and A. D. MacKerell Jr, CHARMM36m: and improved force field for folded and intrinsically disordered proteins. Nature Methods, 2017, 14, 7173.

${ }^{28}$ L. Duan, T. Zhu, C. Ji, Q. Zhanga and J. Z. H. Zhang, Direct folding simulation of helical proteins using an effective polarizable bond force field. Phys. Chem. Chem. Phys., 2017, 19, 15273-15284.

29 T. Frembgen-Kesner, C. T. Andrews, S. Li, N. A. Ngo, S. A. Shubert, A. Jain, O. J. Olayiwola, M. R. Weishaar and A. H. Elcock, Parametrization of Backbone Flexibility in a Coarse-Grained Force Field for Proteins (COFFDROP) Derived from All-Atom Explicit-Solvent Molecular Dynamics Simulations of All Possible Two-Residue Reptides. J. Chem. Theor. Comp., 2015, 11(5), 234154. 
${ }^{30}$ M. Cheon, I. Chang and C. K. Hall, Extending the PRIME model for protein aggregation to all 20 amino acids. Proteins: Str. Func. Bioinf., 2010, 78(14), 29502960.

31 V. A. Wagoner, M. Cheon, I. Chang, C. K. Hall, Computer simulation study of amyloid fibril formation by palindromic sequences in prion peptides. Proteins: Str. Func. Bioinf., 2011, 79, 2132-2145.

32 A. B. Poma, M. Cieplak and P. E. Theodorakis, Combining the MARTINI and Structure-Based Coarse-Grained Approaches for the Molecular Dynamics Studies of Conformational Transitions in Proteins. J. Chem. Theory Comput., 2017, 13(3), 1366-1374.

33 D. De Sancho and R. B. Best, Modulation Of An IDP Binding Mechanism And Rates By Helix Propensity And Non-Native Interactions: Association Of Hif1 $\alpha$ With CBP. Mol. BioSyst., 2012, 8(1), 256-267.

${ }^{34}$ D. Ganguly, W. Zhang and J. Chen, Electrostatically Accelerated Encounter And Folding For Facile Recognition Of Intrinsically Disordered Proteins. PLoS Comp. Biol., 2013, 9(11), e1003363.

${ }^{35}$ M. Enciso and A. Rey, Improvement of Structure-Based Potentials for Protein Folding by Native and Nonnative Hydrogen Bonds. Biophys. J., 2011, 101(6). 147482.

${ }^{36}$ N. L. Dawson, T. E. Lewis, S. Das, J. G. Lees, D. Lee, P. Ashford, C. A. Orengo and I. Sillitoe, CATH: an expanded resource to predict protein function through structure and sequence. Nucl. Acids Res., 2016, 45, D289-D295.

37 T. X. Hoang, A. Trovato, F. Seno, J. R. Banavar and A. Maritan, Geometry and symmetry presculpt the free-energy landscape of proteins. Proc. Natl. Acad. Sci., 2004, (USA) 101, 7960-7964.

38 N.-V. Buchete, J. E. Straub and D. Thirumalai, in Coarse-Graining of Condensed Phase and Biomolecular System, ed. G. A. Voth, CRC Press, Boca Raton, 2009. ch. 10, 141-156.

39 M. Enciso and A. Rey, A refined hydrogen bond potential for flexible protein models. J. Chem. Phys., $2010,132,235102$.

40 N. B. Hung, D.-M. Le and T. X. Hoang, Sequence dependent aggregation of peptides and fibril formation. J. Chem. Phys., 2017, 147, 105102.

41 The UniProt Consortium, UniProt: the universal protein knowledgebase. Nucleic Acids Res., 2017, 45, D158-D169.

42 P. R. Shewry, N. G. Halford, P. S. Belton and A. S. Tatham, The structure and properties of gluten: an elastic protein from wheat grain. Phil. Trans. Roy. Soc. B, 2002, 357, 133-142.

43 H. Wieser, Chemistry of gluten proteins. Food Microbiol., 2007, 24, 115-119.

44 E. A. Bayer, J. P. Belaich, Y. Shoham ancd R. Lamed, Annu. Rev. Microbiol. 2004, 58, 521-554.

45 B. Różycki, M. Cieplak M. Czjzek, Large conformational fluctuations of the multi-domain Xylanase Z of Clostridium thermocellum. J. Struct. Biol., 2015, 191, 68-75.

46 B. Różycki, P.-A. Cazade, S. O’Mahony, D. Thompson and M. Cieplak, The length but not the sequence of peptide linker modules exerts the primary influence on the conformations of protein domains in cellulosome multi-enzyme complexes. Phys. Chem. Chem. Phys., 2017, 19, 21414-21425.

47 M. Sikora, J. I. Sułkowska and M. Cieplak, Mechanical strength of 17134 model proteins and cysteine spliknots. PLoS Comp. Biol., 2009, 5, e1000547.

48 J. I. Sułkowska and M. Cieplak, Mechanical stretching of proteins a theoretical survey of the Protein Data Bank. J. Phys.: Condens. Matter, 2007, 19283201.

49 J. I. Sułkowska and M. Cieplak, Selection of optimal variants of Go-like models of proteins through studies of stretching. Biophys. J., 2008, 95, 3174-3191.

50 A. B. Poma, M. Chwastyk, and M. Cieplak, Polysaccharide-protein complexes in a coarse-grained model. J. Phys.Chem. B., 2015, 119, 12028-12041.

51 K. Wołek, Á. Gómez-Sicilia, and M. Cieplak, Determination of contact maps in proteins: a combination of structural and chemical approaches. J. Chem. Phys., 2015, 143, 243105.

52 J. Tsai, R. Taylor, C. Chothia and M. Gerstein, The packing density in proteins: Standard radii and volumes. J. Mol. Biol., 1999, 290, 253-266.

${ }^{53}$ G. Settanni, T. X. Hoang, C. Micheletti and A. Maritan, Folding pathways of prion and doppel. Biophys. J., 2002, 83, 3533-3541.

54 J. Gu, F. Bai, H. Li and X. Wang, A generic force field for protein coarse-grained molecular dynamics simulations. Int. J. Mol. Sci., 2012, 13, 14451-14469.

55 H. M. Berman, J. Westbrook, Z. Feng, G. Gilliland, T. N. Bhat, H. Weissig, I. N. Shindyalov and P. E. Bourne. The Protein Data Bank. Nucl. Acids Res., 2000, 28, 235-242; www.rcsb.org.

56 A. Kolinski, Protein modeling and structure prediction with a reduced representation. Acta Biochim Pol., 2004, 51(2). 349-71.

57 D. G. Covell, and R. L. Jernigan, Conformation of folded proteins in restricted spaces. Biochem., 1990, 29, $3287-94$.

${ }^{58}$ C. Micheletti, F. Seno, J. R. Banavar and A. Maritan, Learning effective amino acid interactions through iterative stochastic techniques. Proteins Struct. Funct. Genet., 2001, 42, 422-31.

59 M. Cieplak, N.S. Holter, A. Maritan and J. R. Banavar, Amino acid classes and the protein folding problem. J. Chem. Phys., 2001, 114, 1420.

60 A. Korkut and W. A. Hendrickson, A Force Field For Virtual Atom Molecular Mechanics Of Proteins. Proc. Natl. Acad. Sci. (USA), 2009, 106(37), 15667-15672.

61 M. Qin, W. Wang and D. Thirumalai. Protein Folding Guides Disulfide Bond Formation. Proc. Natl. Acad. Sci. (USA), 2015, 112(36), 11241-11246.

62 A. H. Mao, S. L. Crick, A. Vitalis, C. L. Chicoine and R. V. Pappu, Proc. Natl. Acad. Sci. (USA), 2010, 107, 8183-8188.

63 P. Debye and E. Hueckel, Zur Theorie der Elektrolyte. I. Gefrierpunktserniedrigung und verwandte Erscheinungen, Phys. Zeitschrift, 1923, 24, 185-206. 
64 A. Ghavani, E. van der Giessen and P. R. Onck, Coarse-grained potentials for local interactions in unfolded proteins. J. Chem. Theor. Comp., 2013, 9, 432-440.

${ }^{65}$ T. Veitshans, D. Klimov and D. Thirumalai, Protein folding kinetics: time scales, pathways and energy landscapes in terms of sequence-dependent properties. Folding Des., 1997, 2, 1-22.

66 M. P. Allen D. J. Tildesley, Computer simulation of liquids, Oxford University Press, New York, 1987.

${ }^{67}$ K. Wołek and M. Cieplak, Criteria for folding in structure-based models of proteins. J. Chem. Phys., 2016, 144, 185102.

68 B. Różycki and M. Cieplak, Stiffness of the C-terminal disordered linker affects the geometry of the active site in endoglucanase Cel8A. Mol. BioSyst., 2016, 12, 3589-3599.

69 M. Wojciechowski, Á. Gómez-Sicilia, M. Carrión-Vázquez and M. Cieplak, Unfolding knots by proteasome-like systems: simulations of the behavior of folded and neurotoxic proteins. Mol. BioSyst., 2016, 12, 2700-2712.

70 J. Petruska, M. J. Hartenstine and M. F. Goodman, Analysis of strand slippage in DNA polymerase expansions of CAG/CTG triplet repeats associated with neurodegenerative disease. J. Biol. Chem., 1998, 273, 5204-5210.

71 R. H. Walters and R. M. Murphy, Examining Polyglutamine Peptide Length: A Connection between Collapsed Conformations and Increased Aggregation. J. Mol. Biol., 2009, 393(4), 978992.

72 M. Enciso, C. Schütte and L. Delle Site. pH-Dependent Coarse-Grained Model of Peptides. Soft Matter, 2013, 9(26), 6118-6127.

${ }^{73}$ W. L. Jorgenson, J. Chandrasekhar, J. D. Madura, R. W. Impey and M. L. Klein, Comparison of simple potential functions for simulating liquid water. J. Chem. Phys., 1983, 79, 926-935.

74 P. Palencar T. Bleha, Molecular dynamics simulations of the folding of poly(alanine) peptides. J. Mol. Model., 2011, 17, 2367-23745.

75 B. Schuler, E. A. Lipman, P. J. Steinbach, M. Kumke and W. A. Eaton, Polyproline and the ,spectroscopic ruler” revisited with single-molecule fluorescence. Proc. Natl. Acad. Sci. (USA), 2005, 102(8), 27542759.

76 A. Möglich, K. Joder and T. Kiefhaber, End-to-end distance distributions and intrachain diffusion constants in unfolded polypeptide chains indicate intramolecular hydrogen bond formation. Proc. Natl. Acad. Sci. (USA), 2006, 103(33), 1239412399.

77 S. Sanyal, D. F. Coker and D. MacKernan, How flexible is a protein: simple estimates using FRET microscopy. Mol. BioSyst., 2016, 12(4), 29882991.

78 J. Henriques, C. Cragnell and M. Skepo, Molecular Dynamics Simulations of Intrinsically Disordered Proteins: Force Field Evaluation and Comparison with Experiment. J. Chem. Theor. Comp., 2015, 11(7), 34203431.

79 M. Varadi, S. Kosol, P. Lebrun, E. Valentini, M. Blackledge, A. K. Dunker, ... and P. Tompa, PE-DB: A database of structural ensembles of intrinsically disordered and of unfolded proteins. Nucl. Acids Res., 2014, 42(D1), 326335.

${ }^{80}$ P. J. Flory, Spatial Configuration Of Macromolecular Chains. Brit. Polym. J., 1976, 8(1), 1-10.

81 L. X. Peterson, A. Roy, C. Christoffer, G. Terashi and D. Kihara, Modeling disordered protein interactions from biophysical principles. PLoS Comp. Biol., 2017, 13, e1005485.

82 M. Chwastyk, A. Poma Bernaola and M. Cieplak, Statistical radii associated with amino acids to determine the contact map: Fixing the structure of a type I cohesin domain in the Clostridium thermocellum cellulosome. Phys. Biol., 2015, 12, 046002.

${ }^{83}$ R. B. Best, X. Zhu, J. Shim, P. E. M. Lopes, J. Mittal, M. Feig and A. D. MacKerell Jr, Optimization of the additive CHARMM all-atom protein force field targeting improved sampling of the backbone $\phi, \psi$ and side-chain $\chi^{1}$ and $\chi^{2}$ dihedral angles. J. Chem. Theory Comput., 2012, 8, 3257-3273. 


\begin{tabular}{|c|c|c|c|c|c|c|c|c|c|c|}
\hline name & Gly & Pro & Gln & Cys & Ala & Ser & Val & Thr & Ile & $\overline{\text { Leu }}$ \\
\hline type & - & - & $\mathrm{P}$ & $\mathrm{P}$ & $\mathrm{H}$ & $\mathrm{P}$ & $\mathrm{H}$ & $\mathrm{P}$ & $\mathrm{H}$ & $\mathrm{H}$ \\
\hline$s$ & 0 & 0 & 2 & 3 & 3 & 2 & 4 & 2 & 5 & 5 \\
\hline$n_{H}$ & 0 & 0 & 0 & 2 & 1 & 0 & 4 & 0 & 4 & 4 \\
\hline$n_{P}$ & 0 & 0 & 2 & 2 & 1 & 2 & 1 & 2 & 2 & 2 \\
\hline$n_{\max }$ & 23 & 27 & 79 & 11 & 24 & 58 & 9 & 24 & 6 & 13 \\
\hline ID $_{\text {UniProt }}$ & P10275 & Q9NP73 & Q156A1 & Q03751 & Q8R089 & O15417 & P32867 & Q869S5 & Q95US5 & Q80YA8 \\
\hline$\nu$ & 0.56 & 0.93 & 0.61 & 0.48 & 0.57 & 0.54 & 0.46 & 0.56 & 0.51 & 0.49 \\
\hline name & Asn & Asp & Lys & Glu & Met & His & Phe & Arg & Tyr & $\operatorname{Trp}$ \\
\hline type & $\mathrm{P}$ & P- & $\mathrm{P}+$ & P- & $\mathrm{H}$ & $\mathrm{P}$ & $\mathrm{H}$ & $\mathrm{P}+$ & $\mathrm{H}$ & $\mathrm{H}$ \\
\hline$s$ & 2 & 2 & 2 & 2 & 4 & 2 & 6 & 2 & 4 & 5 \\
\hline$n_{H}$ & 0 & 0 & 0 & 0 & 1 & 0 & 4 & 0 & 2 & 4 \\
\hline$n_{P}$ & 2 & 2 & 2 & 2 & 1 & 2 & 2 & 2 & 2 & 3 \\
\hline$n_{\max }$ & 46 & 45 & 11 & 33 & 7 & 23 & 9 & 14 & 6 & 6 \\
\hline ID UniProt & Q54XG7 & Q08438 & Q8CI03 & Q6PCN3 & Q01668 & Q6ZQ93 & Q3S2U2 & P38835 & Q9C5D3 & P86690 \\
\hline$\nu$ & 0.54 & 0.81 & 0.81 & 0.81 & 0.60 & 0.62 & 0.52 & 0.81 & 0.51 & 0.44 \\
\hline
\end{tabular}

TABLE I: Attributes of the residues. The first line (after the name of the residue) indicates the amino acid class. The subscripts + or - indicate whether the amino acids are charged. The second line shows the number $(s)$ of the sidechain contacts that the amino acid can make with all other residues. The third and fourth lines lines show the numbers of sidechain contacts that the amino acids can make with the hydrophobic $\left(n_{H}\right)$ and polar $\left(n_{P}\right)$ residues. The fifth line list the sequential extensions of the longest homopolymeric tracts made with the residue as found in the reviewed part of UniProt database (SProt), with the highest level of certainty $(\mathrm{PE}=1$, see $) \stackrel{41}{ }$ The sixth line gives the corresponding accession code. The seventh line lists the exponent $(\nu)$ for the sequence-length dependence $(n)$ of $R_{g}$. The lines further down are for the remaining 10 residues. His is considered to be uncharged.

\begin{tabular}{|l|l|l|l|l|l|l|l|l|l|l|l|l|l|l|l|l|l|l|}
\hline & Gln & Cys & Ala & Ser & Val & Thr & Ile & Leu & Asn & Asp & Lys & Glu & Met & His & Phe & Arg & Tyr & Trp \\
\hline Gln & 8.63 & & & & & & & & & & & & & & & & & \\
\hline Cys & 7.72 & 7.56 & & & & & & & & & & & & & & & & \\
\hline Ala & 7.39 & 6.97 & 6.42 & & & & & & & & & & & & & & & \\
\hline Ser & 7.64 & 6.97 & 6.53 & 6.65 & & & & & & & & & & & & & & \\
\hline Val & 7.81 & 7.56 & 7.06 & 7.17 & 7.65 & & & & & & & & & & & & & \\
\hline Thr & 7.77 & 7.40 & 6.94 & 6.97 & 7.54 & 7.30 & & & & & & & & & & & & \\
\hline Ile & 8.24 & 7.95 & 7.45 & 7.52 & 8.06 & 7.93 & 8.53 & & & & & & & & & & & \\
\hline Leu & 8.44 & 8.07 & 7.65 & 7.68 & 8.29 & 8.12 & 8.77 & 8.93 & & & & & & & & & & \\
\hline Asn & 8.19 & 7.49 & 7.02 & 7.18 & 7.54 & 7.46 & 7.96 & 8.14 & 7.74 & & & & & & & & & \\
\hline Asp & 8.15 & 7.18 & 6.73 & 6.99 & 7.22 & 7.19 & 7.65 & 7.86 & 7.50 & & & & & & & & & \\
\hline Lys & 8.69 & 7.83 & 7.26 & 7.73 & 7.69 & 7.79 & 8.16 & 8.39 & 8.11 & $\underline{8.59}$ & & & & & & & & \\
\hline Glu & 8.41 & 7.45 & 7.04 & 7.41 & 7.50 & 7.51 & 7.97 & 8.20 & 8.00 & & $\underline{8.90}$ & & & & & & & \\
\hline Met & 8.84 & 8.29 & 7.91 & 7.94 & 8.48 & 8.33 & 8.95 & 9.14 & 8.49 & 8.15 & 8.80 & 8.61 & 9.29 & & & & & \\
\hline His & 8.64 & 8.17 & 7.50 & 7.88 & 7.92 & 7.98 & 8.37 & 8.57 & 8.36 & 8.50 & 8.58 & 8.84 & 8.93 & 8.83 & & & & \\
\hline Phe & 8.95 & 8.50 & 8.17 & 8.24 & 8.69 & 8.58 & 9.11 & 9.34 & 8.65 & 8.51 & 8.79 & 8.75 & 9.55 & 8.98 & 9.73 & & & \\
\hline Arg & 9.26 & 8.24 & 7.99 & 8.27 & 8.31 & 8.50 & 8.76 & 8.98 & 8.87 & 9.12 & & 9.52 & 9.27 & 9.23 & 9.26 & & & \\
\hline Tyr & 9.27 & 8.26 & 8.02 & 8.36 & 8.39 & 8.58 & 8.78 & 9.02 & 8.96 & 9.35 & 9.04 & 9.48 & 9.28 & 9.38 & 9.56 & 9.51 & 9.34 & \\
\hline Trp & 9.58 & 8.95 & 8.65 & 8.75 & 9.22 & 9.14 & 9.57 & 9.79 & 9.11 & 9.10 & 9.21 & 9.48 & 10.02 & 9.66 & 10.17 & 9.82 & 10.08 & 10.85 \\
\hline
\end{tabular}

TABLE II: Average distances, in $\AA$, for the ss contacts as derived from the CATH database. The underlined entries indicate a possibility of forming ionic bridges through the electrostatic attraction. 


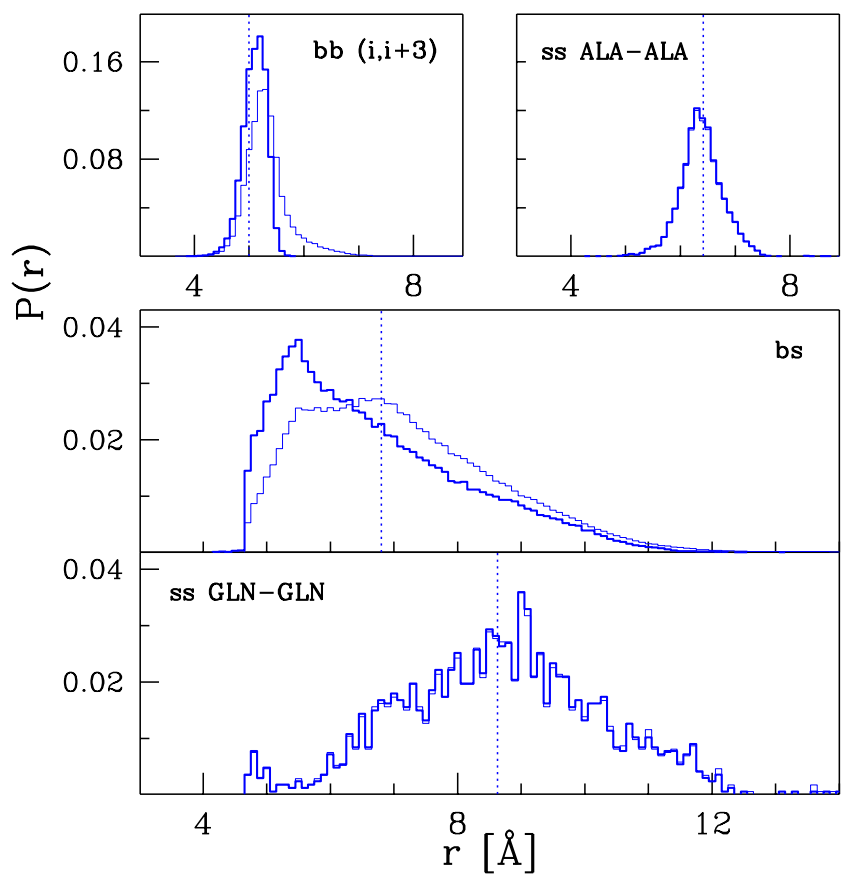

FIG. 1: Examples of the distributions of the $\alpha-\mathrm{C}-\alpha$-C distances in contacts. The distributions are for situations in which not more than one contact is made between the atoms. The thin lines are for all of these situations whereas the thick lines correspond to eliminating contacts which do not satisfy the required directional conditions. The vertical dotted lines indicate the values adopted in our definition of the model. They correspond to the average value calculated based on the thick histograms. The top left panel is for the bb contacts regardless the specificity. Most of them occur in the $i, i+3$ contacts so only those were taken into account. The average distance is about $5.0 \AA$. However, the average distances in other contacts are close in value: $4.7 \AA$ for the $i, i+4$ contacts and $4.8 \AA$ for contacts at all larger sequential distances. The middle panel is for the bs contacts, again regardless the specificity. Almost all of such contacts occur in contacts $i, i+k$, where $k \geq 5$ and only these were included in the distribution. The remaining panels are for the ss contacts for Ala-Ala (the top right panel) and Gln-Gln (the bottom panel). These are determined for all sequential distances combined. Similar panels for all possible distributions corresponding to the ss contacts are shown in the Electronic Supplementary Information (Figs. S1-S3). 

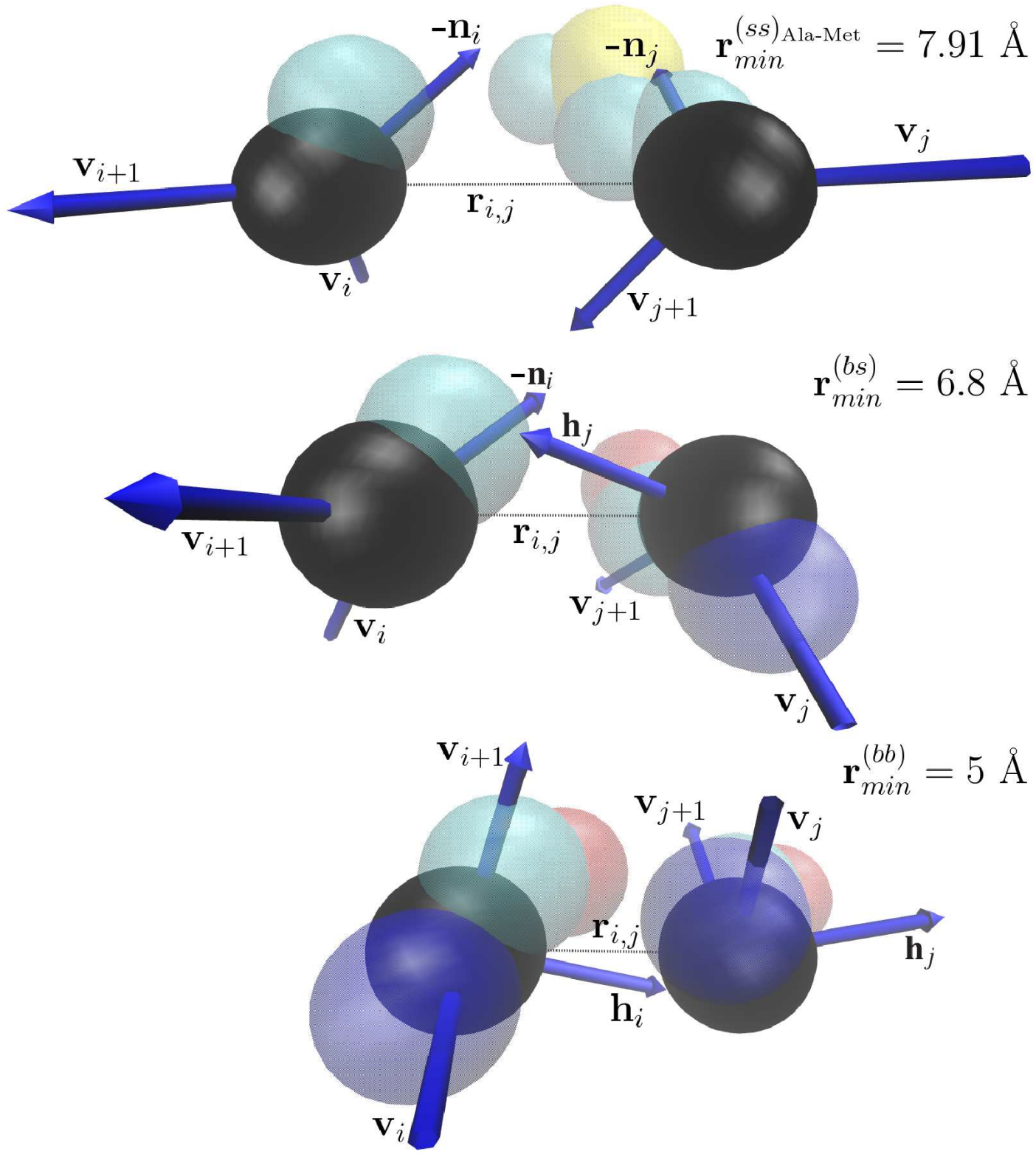

FIG. 2: Examples of contact formation between Ala (the residue on the left) and Met. The distance between the $\alpha$-C atoms (shown as spheres in black) are 7.91, 6.03 and $5.4 \AA$ top to bottom respectively. The top, middle and bottom panels illustrate formation of an ss, bs and bb contacts respectively. The first two panels are obtained in one trajectory of an all-atom simulation (performed using NAMD). The bottom panel shows a fragment of an $\alpha$-helix in the structure PDB:14GS. The $\alpha$-C atoms are shown as black spheres. The other atoms are shown using the CPK coloring scheme. The hydrogen atoms are not shown. The sidechain atoms are represented by semi-transparent spheres. The arrows indicate either vectors $\mathbf{h}$ (for the backbone-involving interactions) or $\mathbf{n}$ (for the sidechain-involving interactions, as indicated. Vector $r_{i, j}$ connecting $\alpha$-C atoms is drawn as a dotted line. The threshold values of $r_{i, j}$, at which a given kind of contact is considered to be setting in, are written on the right. $\mathbf{v}_{i}$ is defined as $\mathbf{r}_{i}-\mathbf{r}_{i-1}$. 


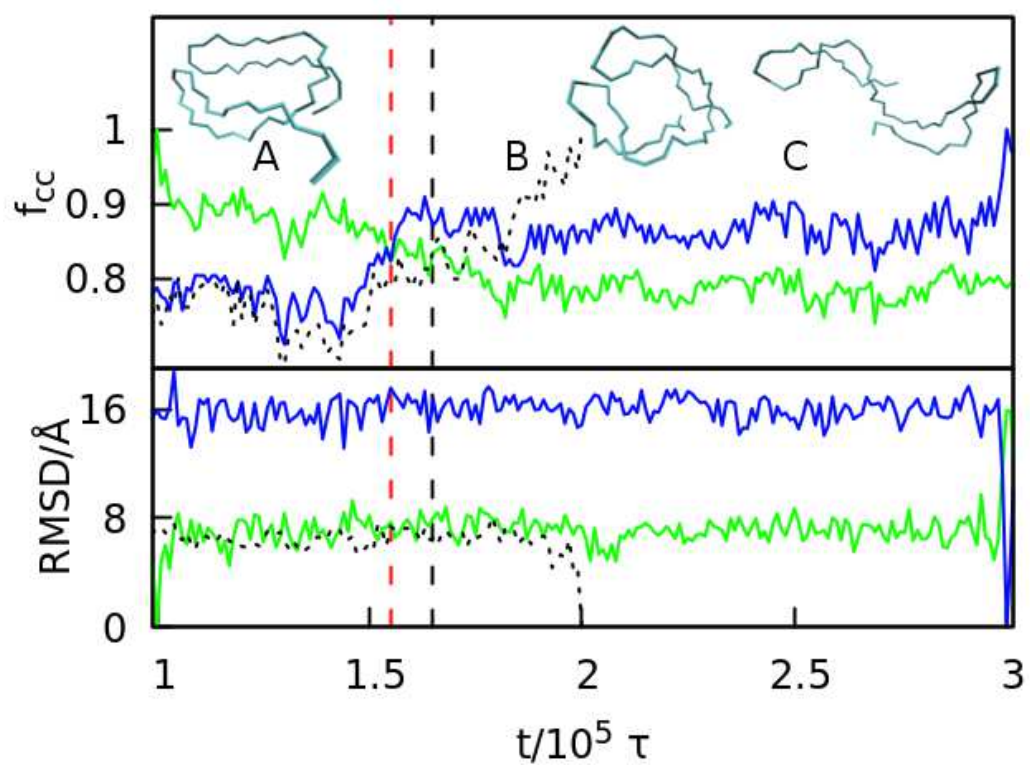

FIG. 3: The time-dependence of $f_{c c}$ (top) and RMSD (bottom) for $\mathrm{Q}_{60}$. It is calculated every $1000 \tau$ with respect to three reference structures obtained at $10^{5} \tau(\mathrm{A}), 2 \cdot 10^{5} \tau\left(\mathrm{B}\right.$, dotted line), and $3 \cdot 10^{5} \tau(\mathrm{C})$ in an example of a trajectory. The reference structures are depicted at the top. The red vertical line indicates a transition from a similarity in $f_{c c}$ between $\mathrm{A}$ and C. The black vertical line: between $\mathrm{A}$ and $\mathrm{B}$.

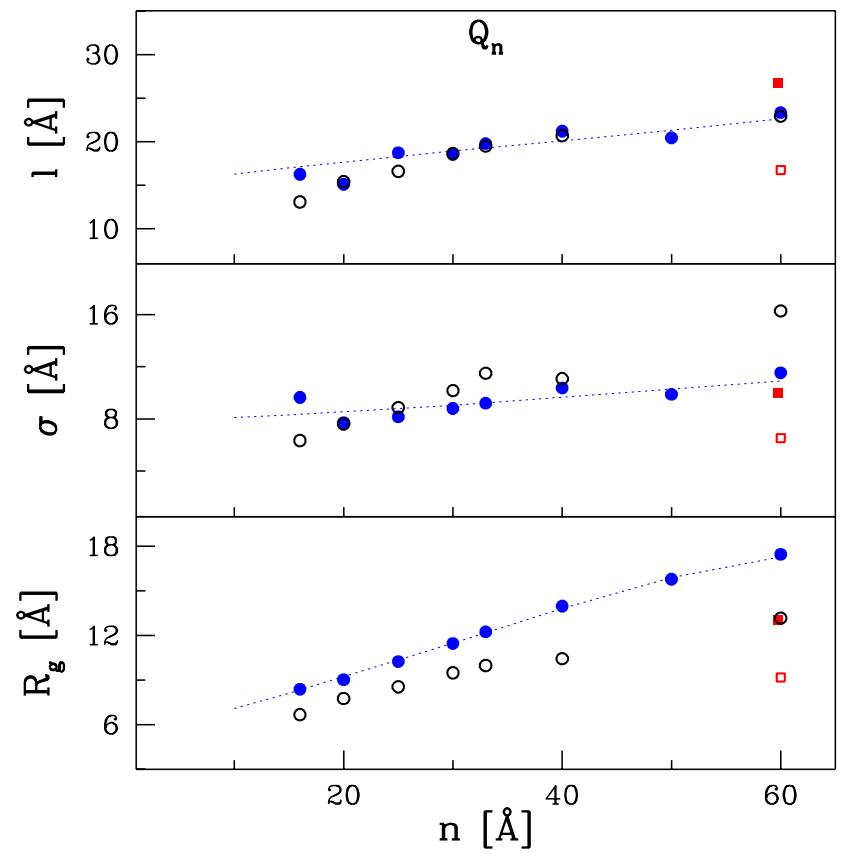

FIG. 4: The results obtained by our IDP coarse-grained model for $\mathrm{Q}_{n}$ (solid blue circles) and $\mathrm{V}_{n}$ (solid red squares) as a function of the number of residues, $n$. The top panel is for $l$, the middle for $\sigma$, and the bottom one for $R_{g}$. The open circles correspond to the results obtained by all-atom simulations ${ }^{24}$ for $\mathrm{Q}_{n}$. The open red squares - only for $n=60-$ correspond to the results obtained by all-atom simulations ${ }^{20}$ for $\mathrm{V}_{60}$. The lines are guides to the eye. The sizes of the symbols are of the order of the error of the mean. 


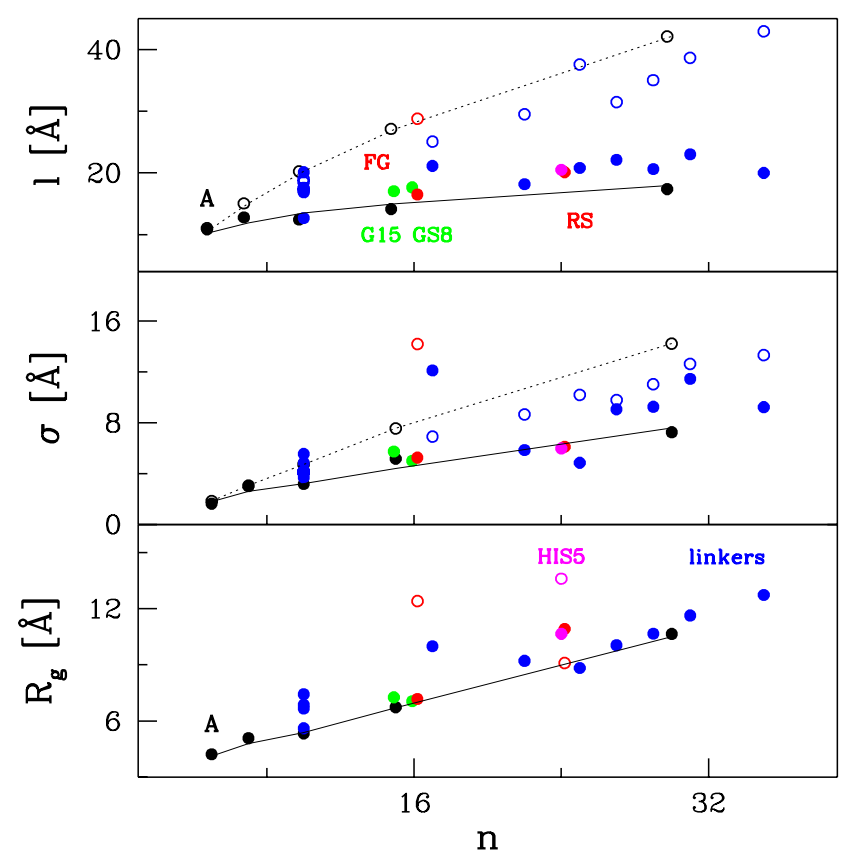

FIG. 5: Similar to Fig. 4 but for other indicated systems. The results obtained by our IDP coarse-grained model are shown using the full circles and those obtained by all-atom simulations - by the open circles. The black data points are for polyalanine. The blue data points - for selected linkers. ${ }^{46}$ The selected linkers are those with $n=10$ and $n>15$. The red data points are for the systems denoted as FG and RS. Note that in the case of FG, the all-atom results are available only for $R_{g}$. The green data points are for G15 and GS8. The magenta data points are for His5.

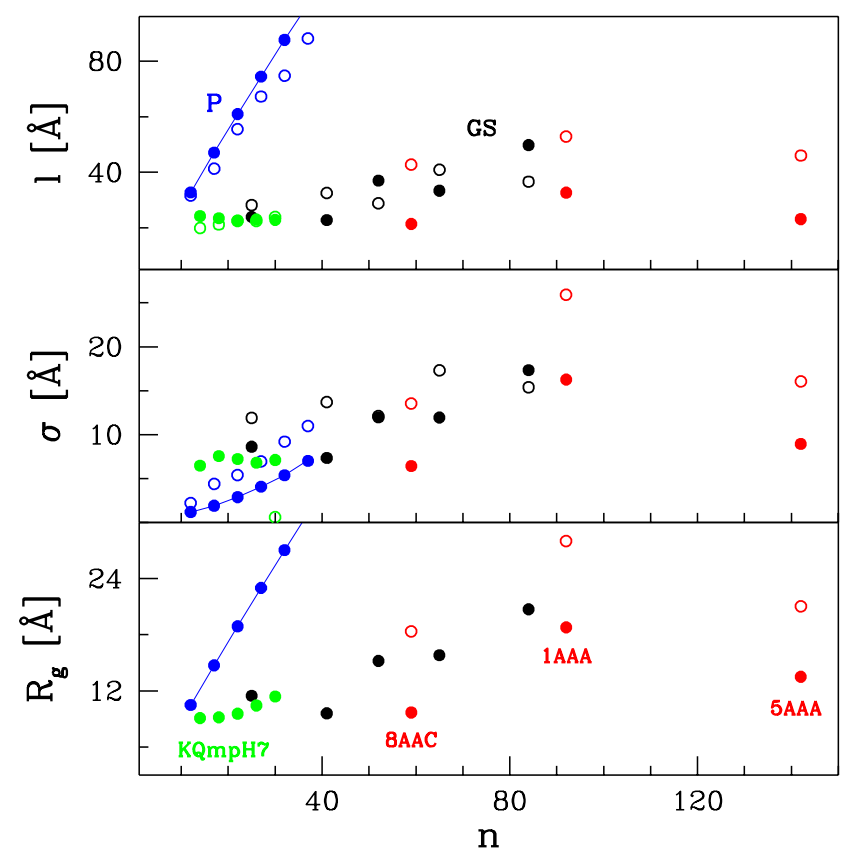

FIG. 6: Similar to Figs. 4 and 5 but now the comparison is to the results obtained experimentally (the open circles). The blue data points are for polyproline. The black data points are for "GS". The red data points are for the three indicated systems from PEDb. 6AAA and 9AAA disagree with the theoretical findings more significantly and are not shown. The green data points are for $\mathrm{KQn}$ at $\mathrm{pH}=7$. 


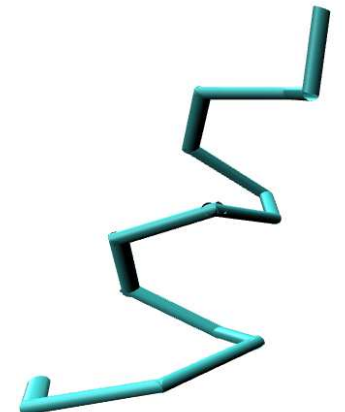

$\mathrm{A}_{15}$

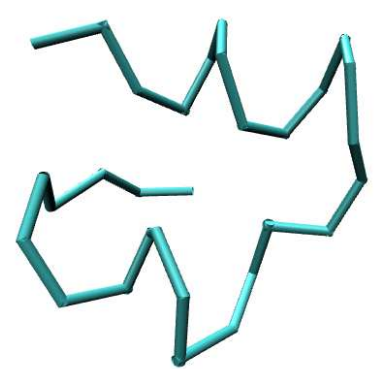

$\mathrm{A}_{30}$
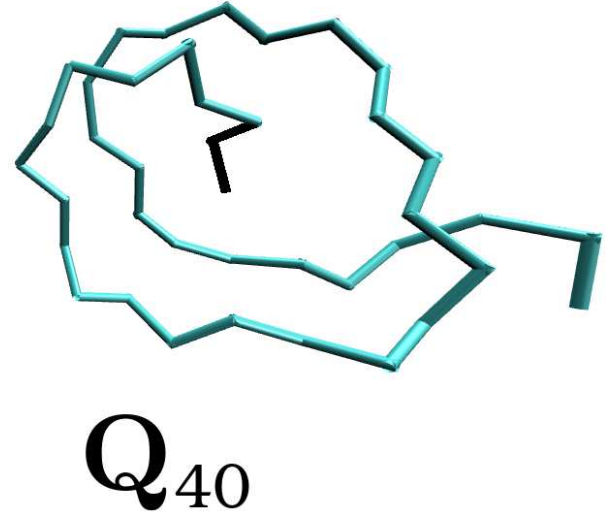

FIG. 7: Examples of conformations of three homopeptides: $\mathrm{A}_{15}$ (left), $\mathrm{A}_{30}$ (middle) and Q40. The latter conformation is knotted. The N-terminal end of $\mathrm{Q}_{40}$ is shown in black. It crosses the loop inwards, forming a shallow knot.

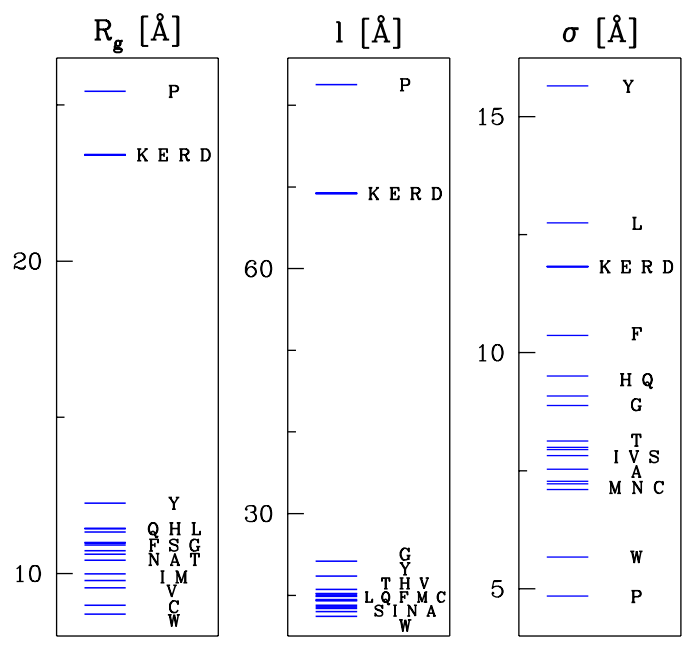

FIG. 8: The geometrical parameters $R_{g}, l$ and $\sigma$ for the homopeptides of length 30. The error of the mean does not exceed 1,3 and $2 \%$ for $R_{g}, l$ and $\sigma$ respectively. The types of the amino acids are indicated in the single letter convention. If we considered the histidine residues to be charged, the parameters would be the same as for K,E,R and D. 


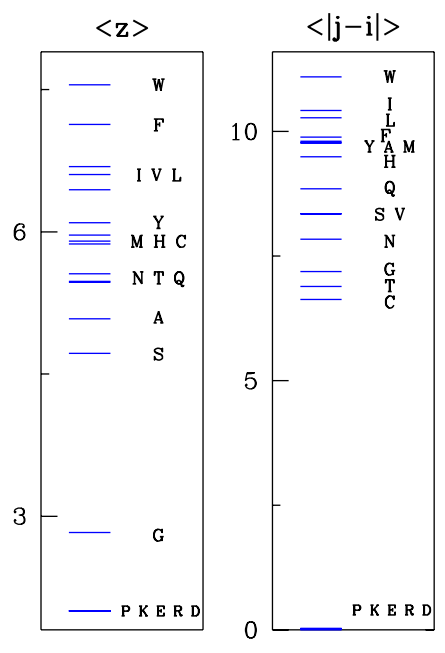

FIG. 9: Similar to Fig. 8 but for the average coordination number and the average sequential distance in the contacts. Contact order is this distance divided by $n$. The error of the mean does not exceed 0.5 and $3.3 \%$ for $\langle z\rangle$ and $\langle|j-i|>$ respectively. 